\title{
Spontaneous Spinal Subdural Hematoma Developed After Weightlifting: A case Report and Review of Literature
}

\author{
JiEun Park, SooMi Lim, Jeong Hyun Yoo*
}

Department of Radiology, Mokdong Hospital, EwhaWomans University School of Medicine, Seoul, Korea.

Email: ${ }^{*}$ yoolee@ewha.ac.kr

Received 5 July 2011; revised 3 August 2011; accepted 23 August 2011.

\begin{abstract}
Spontaneous spinal subdural hematoma is a rare disease. Several predisposing factors are reported including impaired clotting due to therapeutic anticoagulation or hematologic disorder, vascular anomaly, intraspinal tumor, and iatrogenic causes such as lumbar puncture or spinal anesthesia.We report a rare case of spontaneous spinal subdural hematoma developed after weightlifting in a healthy young man treated with conservative treatment.
\end{abstract}

Keywords: Spinal Hematoma; Spinal Subdural Hematoma; Spontaneous Subdural Hematoma

\section{INTRODUCTION}

Spontaneous spinal subdural hematoma (SSDH) is a rare disease entity with little known pathogenesis. It is known to be associated with several predisposing factors including impaired clotting due to therapeutic anticoagulation or hematologic disorder, vascular anomaly, intraspinal tumor, and iatrogenic causes such as lumbar puncture or spinal anesthesia. There is an ongoing controversy on whether surgical or conservative treatment should be preferred in SSDHs. Here, we report a case of spontaneous SSDH with concomitant intracranial hypotension developed after weightlifting, without underlying predisposting factors and treated with conservative treatment.

\section{CASE REPORT}

A 38-year-old man was referred to the emergency room with severe headache and upper back pain. A month before, he had a sudden onset upper back pain while lifting weights and was subsequently treated with physical therapy at a local clinic. He had been weight lifting on regular basis, twice a week for about 3 months so far. There was no history of direct trauma to the upper neck. The pain was not subsided, and new symptoms of head- ache with vomiting aroused several hours before he visited the emergency room. No neurologic abnormalities were found. Brain computed tomography(CT) was within normal limits. However, brain MRI showed diffuse dural thickening and intense meningeal enhancement indicating intracranial hypotension(Figure 1).

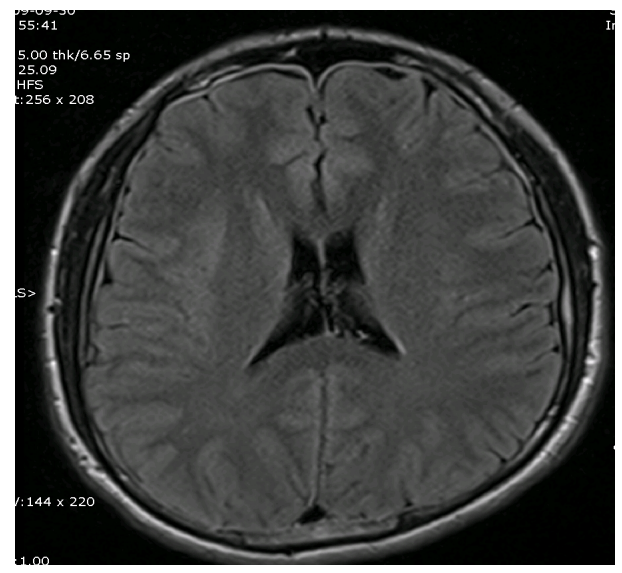

(a)

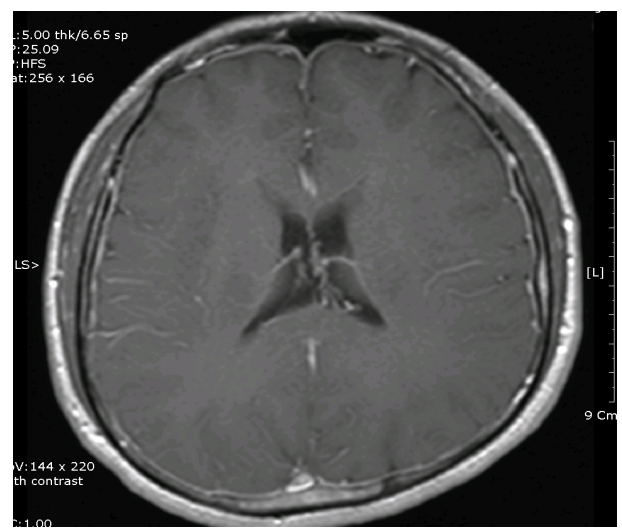

(b)

Figure 1. FLAIR axial image shows diffuse dural thickening (a) and enhanced axial image shows diffusepachymeningeal enhancement (b). 
On admission, his coagulation profiles including platelet count, prothrombin time, and partial thromboplastin time were within normal range. There was no previous history of anticoagulant therapy or hematologic disease, and he had been in good health. He did not take any medication.Cervical spine MRI revealed a well-demarcated ventral subdural hematoma ranging from C6 to T5, mildly compressing the cervical spinal cord (Figure 2). The T1-weighted MR images of the hematoma showedhigh and low signal intensities, and the T2-weighted image showed a mixed pattern of lowand dark signal intensities. Based on these findings, an acute to early subacute stage of spinal SDH was diagnosed.

The patient was prescribed with IV analgesics to reduce headache, absolute bed rest to prevent intracranial hypotension, and IV steroids to prevent intramedullary edema. Initial IV steroids were gradually tapered out a week later, as he did not show any neurologic deficit on subsequent exams. MR images at 2 weeks of conservative treatment showed hematoma resorption with improvement of compressive myelopathy. Follow up MRI at 10 weeks showed complete resolution of the hematoma without residual symptom (Figure 3).

\section{DISCUSSION}

Spontaneous spinal subdural hematoma (SSDH) is a rare disease entity first described by Shiller and coworkers more than 60 years ago [1]. Several predisposing factors are reported to have caused this, including impaired clotting due to therapeutic anticoagulation or hematologic disorder, vascular anomaly, intraspinal tumor, and iatrogenic causes such as lumbar puncture or spinal anesthesia. However, the pathogenesis of spontaneous SSDHs is little understood.

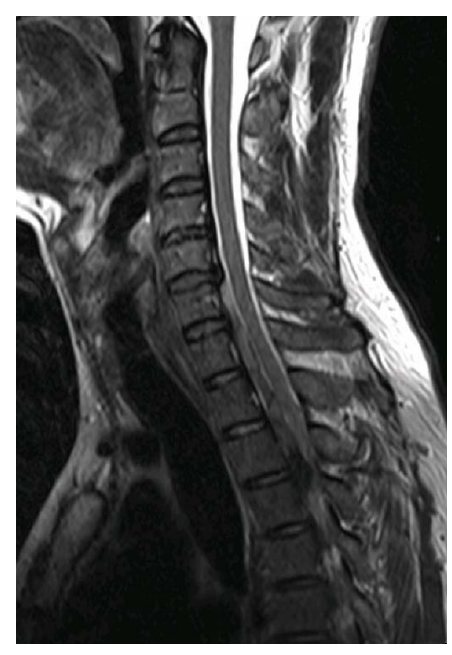

(a)

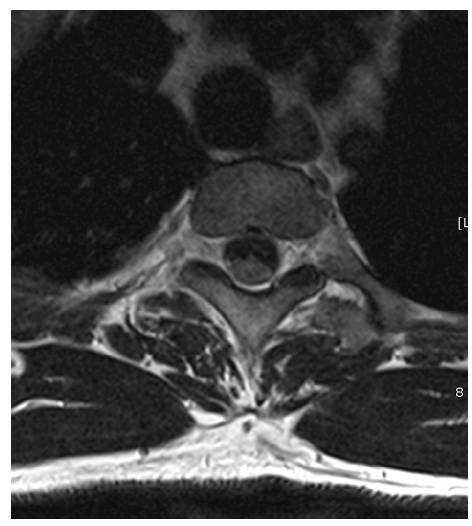

(c)

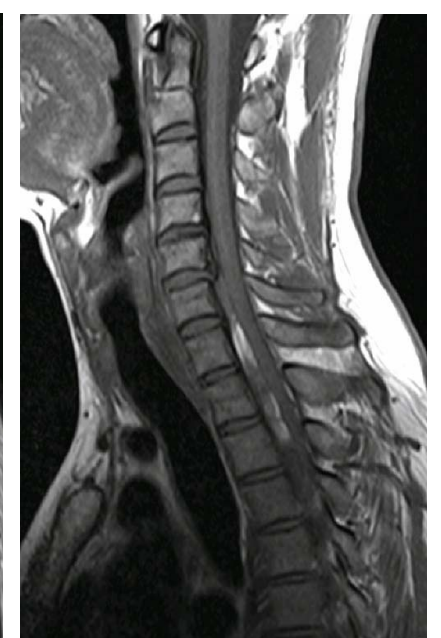

(b)

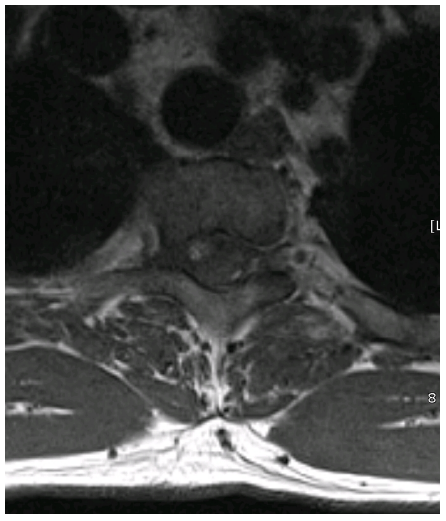

(d)

Figure 2. Spine MR shows spinal subdural hemorrhage anterior to the spinal cord from the C6 to L5 level with iso- and low signal intensity on T2-weighted image (a, c) and high and low- signal intensity on T1-weighted (b,d). Axial image scanned at T2 level reveals the lesion compressing the spinal cord. 


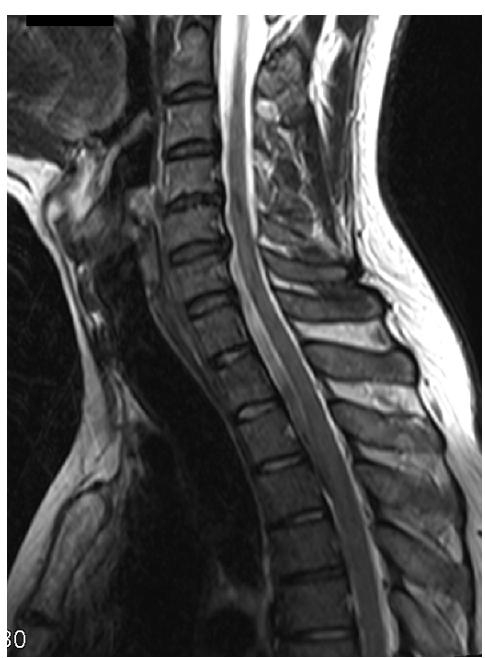

(a)

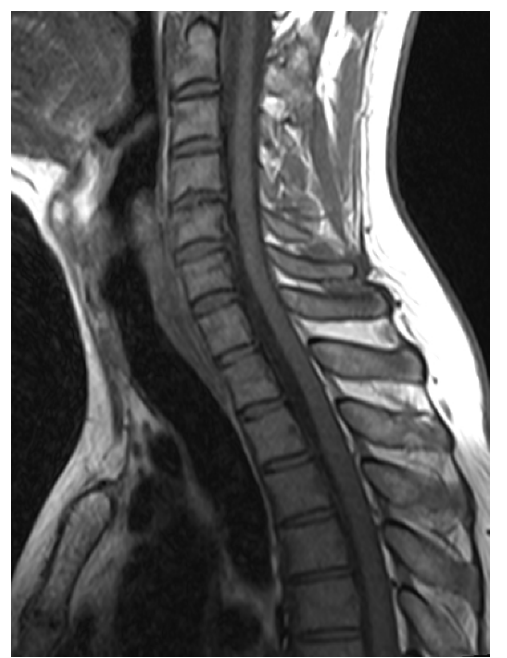

(b)

Figure 3. Follow up MRI at 10 weeks. Sagittal T2-weighted (a) and T1weighted (b) MRI shows resolution of the hematoma.

A commonly accepted hypothesis is the rupture of valvelessradiculomedullary veins that cross the subdural and subarachnoid spaces causing spontaneous SSDHs [2, 3].Additionally, rupture of the small extra-arachnoidal vessels located on the inner surface of the dura also results in subdural hematomas [4]. Rader et al. hypothesized that sudden increase in this intravascular pressure in the spinal subdural space causes disparity between intravascular and extravascular pressure resulting in the tearing of the spinal vessels [5].

Another hypothesis about the origin of spontaneous SSDHs relates to the anatomical structure of the dura mater. The dura mater has two layers, the strong external layer and the fairly weaker inner layer, the latter of which is susceptible to injury. Minor trauma causes tear of the inner dura, and damage to theradiculomedullary veins which cross it. Hung et al. hypothesized that the rising of intracranial pressure increasesthe shearing force between spinal subdural and subarachnoid spaces so that the inner dura may tear and bleed [6].

As far as I concerned, it is the rare case report that weightlifting caused spontaneous spinal subdural hematoma. We presumed the excessive Valsalva maneuver developed during the weightlifting that caused increaseing the intravascular pressure with/without direct minor trauma to the spinal inner dura, whichfinally resulted in spontaneous spinal subdural hematomas. In this patient, SSDH was also accompanied with intracranial hypotension. This intracranial hypotension associated with SSDH may be a result of CSF leakage at the site of the hemorrhage, although a reverse hypothesis can be possible that intracranial hypotension induces cerebro-spinal hypotension leading to SSDH.
SSDH occursmost often in the thoracic level with the initial symptomsof sudden onset back pain radiating limb or trunk. Neurologic deficits are also observed with variable degrees of motor, sensory, and autonomic disturbance, but the symptom onset to neurologic deficit shows variable time interval. If the patient with anticoagulant therapy history have abrupt onset of spinal cord compressing symptom or sign, SSDH must be considered. Prompt diagnostic work-up required for the correct diagnosis. MRI is the preferred method showing usually ventral located space-occupying hematoma.

Our patient has no history such as coagulation therapy or other presumptive history suggestive SSDH. Although the initial symptom was the abrupt posterior neck pain, the initial symptomwas skipped by the patients.If the diagnosis was early, spinal MRI would show hyperacute or acute stage of SSDH, but our case showed delayed hematoma with subacutestage.

There is an ongoing controversy on whether surgical or conservative treatment should be preferred in SSDHs. As there is no underlying pathology to undergone surgery, therapy is targeted to decrease the hematoma in spontaneous SSDHs. Several authors proposed that conservative management may be indicated when the severity of neurologic symptoms is less than grade $\mathrm{D}$ or better on the Frankel grading scale [10-12]. Since the patient did not show definite neurologic deficit, conservative treatment was the mode of choice, and complete resolution of the hematoma was obtained. We agree that prompt surgical evacuation should be considered to patients with severe neurologic deficit, before irreversible damage is done to the spinal cord. However, it is recommended to consider conservative management when 
neurologic symptoms are mild or the symptoms improve quickly to the treatment of spinal edema.

\section{CONCLUSIONS}

Several predisposing factors are reported to have caused this spontaneous spinal subdural hematoma (SSDH). The weightlifting practical exercise can be a one of cause of developing the spontaneous SSDH which can associate with intracranial hypotension.

\section{REFERENCES}

[1] Dickerman, R.D., McConathy, W.J., Smith, G.H., East, J.W. and Rudder, L. (2000) Middle cerebral artery blood flow velocity in elite power athletes during maximal weight-lifting. Resarch on Neurology, 22, 337-340.

[2] Dickerman, R.D. and Morgan, J. (2005) Pathogenesis of subdural hematoma in healthy athletes: Postexertional intracranial hypotension? Acta Neurochirurgica (Wien), 147, 349-350. doi:10.1007/s00701-004-0476-0

[3] Hung, K.S., Lui, C.C., Wang, C.H., Wang, C.J. and Howng, S.L. (2002) Traumatic spinal subdural hematoma with spontaneous resolution. Spine (PhilaPa 1976), 27, E534-E538.

[4] Kakitsubata, Y., Theodorou, S.J., Theodorou, D.J., Miyata, Y., Ito, Y., Yuki, Y., Honbu, K. and Maehara, T. (2010) Spontaneous spinal subarachnoid hemorrhage associated with subdural hematoma at different spinal levels. Emergency Radiology, 17, 69-72. doi:10.1007/s10140-008-0792-4

[5] Kirsch, E.C., Khangure, M.S., Holthouse, D. and
McAuliffe, W. (2000) Acute spontaneous spinal subdural haematoma: MRI features. Neuroradiology, 42, 586-590. doi:10.1007/s002340000331

[6] Kyriakides, A.E., Lalam, R.K. and El Masry, W.S. (2007) Acute spontaneous spinal subdural hematoma presenting as paraplegia: A rare case. Spine (PhilaPa 1976), 32, E619-E622

[7] Maeda, M., Mochida, J., Toh, E., Nishimura and K., Nomura, T. (2001) Nonsurgical treatment of an upper thoracic spinal subdural hemorrhage. Spinal Cord, 39, 657-661. doi:10.1038/sj.sc.3101219

[8] Ozdemir, O., Calisaneller, T., Yildirim, E., Caner, H. and Altinors, N. (2008) Acute spontaneous spinal subdural hematoma in a patient with bilateral incarcerated inguinal hernia. Joint Bone Spine, 75, 345-347. doi:10.1016/j.jbspin.2007.05.019

[9] Payer, M. and Agosti, R. (2010) Spontaneous acute spinal subdural hematoma: spontaneous recovery from severe paraparesis-case report and review. Acta Neurochirurgica (Wien), 152, 1981-1984. doi:10.1007/s00701-010-0758-7

[10] RADER, J.P. (1955) Chronic subdural hematoma of the spinal cord: Report of a case. The New England Journal of Medicine, 253, 374-376. doi:10.1007/s00701-010-0758-7

[11] Schiller, F., Neligan, G. and Budtz-Olsen, O. (1948) Surgery in haemophilia; a case of spinal subdural haematoma producing paraplegia. Lancet, 2, 842-845. doi:10.1016/S0140-6736(48)91427-5

[12] Zeidan, A., Farhat, O., Maaliki H and Baraka A. (2010) Does postdural puncture headache left untreated lead to subdural hematoma? Case report and review of the literature. Middle East Journal of Anesthesiology, 20, 483-492. 